\title{
Erratum: "Revisit of Nonlinear Landau Damping for Electrostatic Instability Driven by Blazar-induced Pair Beams" (2019, ApJ, 873, 10)
}

\author{
P. J. Deka ${ }^{1}$ (D), M. Pohl ${ }^{1,2}$, S. Vafin ${ }^{1}$, and A. Bohdan ${ }^{2}$ \\ ${ }^{1}$ Institute for Physics and Astronomy, University of Potsdam, D-14476 Potsdam, Germany \\ 2 DESY, Platanenallee 6, D-15738 Zeuthen, Germany \\ Received 2019 September 8; published 2019 October 4
}

In the published article, the calculation of the power losses of the beam did not take into account the equipartition of beam energy into kinetic energy and electrostatic energy of the plasma waves. We correct for this inaccuracy in this erratum: the power losses of the beam would increase by a factor of two. Equation (17) in the published article changes to

$$
P(t)=8 \pi \int \omega_{i} W(\boldsymbol{k}, t) k_{\perp} d k_{\perp},
$$

where $W_{b}(t=0)=\left\langle\gamma_{b}\right\rangle n_{b} m_{e} c^{2}$ is the initial beam energy density, and $\alpha$ is the fraction of initial beam energy lost at time $\tau(\alpha)$. Using Equation (17), we recalculate the power losses of the beam for three different IGM temperatures. The new relaxation times, summarized in Table 1, are reduced by almost a factor of two. Similar differences in the relaxation times are obtained when the effect of particle collisions is taken into account (Table 2).

This additional factor of two brings about noticeable changes in the power loss spectrum (Figures 8 and 12 in the published article). The corrected versions of Figures 8 and 12 are shown here. Similarly, the equation for power loss in the 3D model (Equation (40)) changes to

$$
P(t)=8 \pi \int \omega_{i} W(\boldsymbol{k}) k_{\perp} d k_{\perp} d k_{\|}
$$

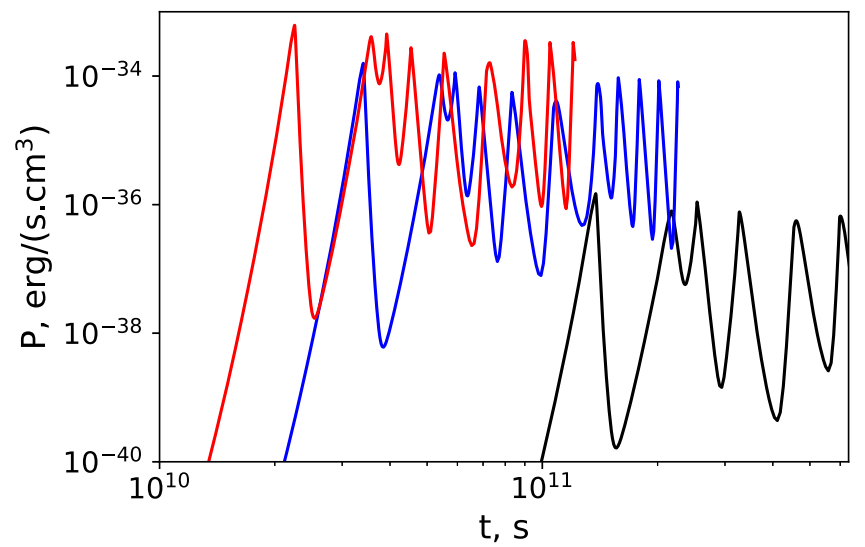

Figure 8. Power losses of the pair beam, Equation (17), for cases 1 (black), 2 (blue), and 3 (red).

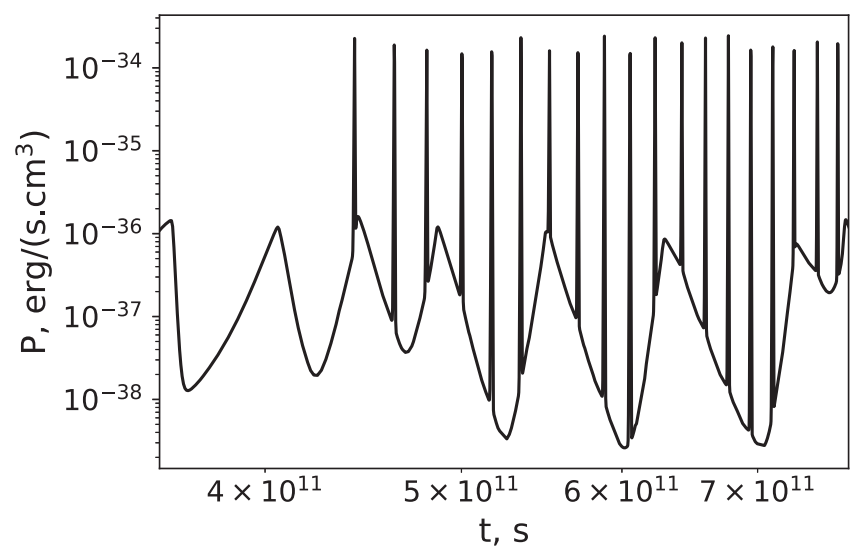

Figure 12. Power losses of the pair beam, Equation (17), for case 4. 
Table 1

Simulation Parameters and Relaxation Time

\begin{tabular}{lccr}
\hline \hline Case & $n_{e},\left(\mathrm{~cm}^{-3}\right)$ & $T_{e},(\mathrm{eV})$ & $\tau_{\text {rel }},(\mathrm{s})$ \\
\hline 1 & $10^{-7}$ & 1 & $3.6 \times 10^{15}$ \\
2 & $10^{-7}$ & 5 & $3.5 \times 10^{13}$ \\
3 & $10^{-7}$ & 8 & $1 \times 10^{13}$ \\
\hline
\end{tabular}

Table 2

Simulations Including Particle Collisions

\begin{tabular}{lccr}
\hline \hline Case & $n_{e},\left(\mathrm{~cm}^{-3}\right)$ & $T_{e},(\mathrm{eV})$ & $\tau_{\text {rel }},(\mathrm{s})$ \\
\hline 4 & $10^{-7}$ & 1 & $1.6 \times 10^{14}$ \\
5 & $10^{-7}$ & 0.8 & $1.25 \times 10^{14}$ \\
6 & $10^{-7}$ & 0.3 & $4.5 \times 10^{13}$ \\
\hline
\end{tabular}

It is to be noted that the inclusion of the additional factor of two for determining the power loss of the beam hardly has any effect on the final results.

\section{ORCID iDs}

P. J. Deka (1) https://orcid.org/0000-0002-6027-9555 